\title{
SOME ELEMENTS IN THE STABLE HOMOTOPY OF SPHERES
}

\author{
WEN-HSIUNG LIN ${ }^{1}$
}

ABSTRACT. An infinite family in ${ }_{2} \pi_{*}^{*}$ at Adams filtration 4 is constructed.

Let $A$ denote the mod 2 Steenrod algebra. There is a spectral sequence $\left\{E_{r}^{s, t}\right\}$ which converges to the 2-primary component of the stable homotopy groups of spheres ${ }_{2} \pi_{*}^{s}$ and has

$$
E_{2}^{s, t} \cong \operatorname{Ext}_{A}^{s, t}\left(\mathbf{Z}_{2}, \mathbf{Z}_{2}\right) \text {. }
$$

This is known as the mod 2 Adams spectral sequence [1].

Let $h_{i} \in \operatorname{Ext}_{A}^{1,2^{i}}\left(\mathbf{Z}_{2}, \mathbf{Z}_{2}\right)$ be the class corresponding to the generator $\mathrm{Sq}^{2^{i}} \in A$ as described by Adams in [2]. J. P. May shows in [12] that $\operatorname{Ext}_{A}^{3,22}\left(\mathbf{Z}_{2}, \mathbf{Z}_{2}\right) \cong \mathbf{Z}_{2}$ generated by an element called $c_{1}$. D. M. Davis shows in [9] that $c_{1} h_{i} \neq 0$ in $\operatorname{Ext}_{A}^{4} \cdot 2^{\prime}+11\left(\mathbf{Z}_{2}, \mathbf{Z}_{2}\right)$ for $i \geqslant 11$. In this paper we prove

THEOREM 1. For $i \geqslant 11, c_{1} h_{i}$ survives the Adams spectral sequence and so detects homotopy elements in $\pi_{2^{\prime}+18}^{s}$.

This establishes an infinite family in ${ }_{2} \pi_{*}^{s}$ at Adams filtration 4 . This family cannot be constructed from elements of lower Adams filtrations so far exposed [7, 11].

Using May spectral sequence [12] one can show that $c_{1} h_{i} \neq 0$ for $5 \leqslant i \leqslant 10$ also, and our result is true for these cases too. The case $i=5$ was published by M. C. Tangora in [14] and other cases have not yet formally published.

The proof is based on Mahowald's technique in [11], where he proves $h_{1} h_{i}$ detects homotopy elements for $i \geqslant 3$. For $a, b \in \mathbf{Z}$ with $b<a$ there is a spectrum $P_{b}^{a}$ which, when $b>0$, is the suspension spectrum of stunted real projective space $\mathbf{R} P^{a} / \mathbf{R} P^{b-1}$. These can be defined as Thom spectra or using James periodicity as in [3]. Let $S^{n}$ denote the sphere spectrum in stable dimension $n$. In the following, the cohomology groups have mod 2 coefficients.

Proposition 2. Suppose $i \geqslant 1$. Then

(a) There is a map $f: P_{-2^{i-1}-1}^{-2} \rightarrow S^{-2^{i}-1}$ such that, in the mapping cone $X=S^{-2^{i}-1}$ $\cup_{f} C P_{-2^{i-1}-1}^{-2}$, the Steenrod operation

$$
\mathrm{Sq}^{2^{i}}: H^{-2^{i}-1}(X)=\mathbf{Z}_{2} \rightarrow H^{-1}(X)=\mathbf{Z}_{2}
$$

is nonzero.

Received by the editors June 27, 1984 and, in revised form, August 8, 1984.

1980 Mathematics Subject Classification. Primary 55Q45, 55T15.

${ }^{1}$ Partially supported by a grant from the National Science Foundation.

(1985 American Mathematical Society $0002-9939 / 85 \$ 1.00+\$ .25$ per page 
(b) There is a map $g: S^{17} \rightarrow P_{-2^{i-1}-1}^{-2}$ such that the composite

$$
S^{17} \stackrel{g}{\rightarrow} P_{-2^{i-1}-1}^{-2} \stackrel{p}{\rightarrow} S^{-2}
$$

is detected by $c_{1}$ where $p$ is the collapsing map.

This implies that $c_{1} h_{i}$ are permanent cycles in the Adams spectral sequence (in deducing this, one needs the fact that in $X$ primary operations from the bottom cell to other cells of dimensions $\leqslant-2^{i-1}-1$ are trivial and this follows by dimensional reasons). Since $\operatorname{Ext}_{A}^{s, t}\left(\mathbf{Z}_{2}, \mathbf{Z}_{2}\right)=0$ for $s \leqslant 2$ and $t-s=2^{i}+19$ [2], $c_{1} h_{i}$ are not boundaries. This proves Theorem 1 .

The result 2(a) is not new; it is primarily due to Mahowald [11]. A detailed proof of it can be found in [8]. For completeness we sketch the proof as follows.

Consider the double loop space $\Omega^{2} S^{3}$ where $S^{3}$ now stands for the 3-sphere. V. P. Snaith shows in [13] that there is a stable splitting

$$
\Omega^{2} S_{+}^{3} \simeq \bigvee_{k \geqslant 0} D_{k}
$$

Here $\Omega^{2} S_{+}^{3}$ denotes $\Omega^{2} S^{3}$ with a disjoint base point, $D_{0}=S^{0}$ (two points) and for $k \geqslant 1$

$$
D_{k}=F\left(\mathbf{R}^{2}, k\right)+\wedge_{\Sigma_{k}}(\underbrace{S^{1} \wedge \dot{\cdots} \wedge S^{1}}_{k}),
$$

where $F\left(\mathbf{R}^{2}, k\right)$ is the configuration space of $k$-tuples of distinct points in $\mathbf{R}^{2}$. In [11] Mahowald showed that for $k \geqslant 1$

(1) $H^{*}\left(D_{k}\right) \cong A / A\left\{\chi\left(\mathrm{Sq}^{i}\right): i>[k / 2]\right\}$ generated by a class $u_{k} \in H^{k}\left(D_{k}\right)$,

and conjectured that, at prime $2, D_{k}$ had the stable homotopy type of the BrownGitler spectrum $B([k / 2])$ [5]. Here $\chi: A \rightarrow A$ is the canonical antiautomorphism of $A$. Mahowald's conjecture was later proved by Brown and Peterson in [6]. BrownGitler spectra $B([k / 2])$ are characterized by (1) together with an additional property which will not be described here (see [6]). A corollary of this property is the following:

Let $M$ be a closed manifold of dimension $n \leqslant k$ and let $T\left(\nu_{M}\right)$ be the Thom spectrum of the stable normal bundle $\nu_{M}$ of $M$ with Thom class $U \in H^{*}\left(T\left(\nu_{M}\right)\right)$ lying in dimension zero. Then $\exists$ a map $\phi: \Sigma^{k} T\left(\nu_{M}\right) \rightarrow D_{k}$ such that $\phi^{*}\left(u_{k}\right)=U$.

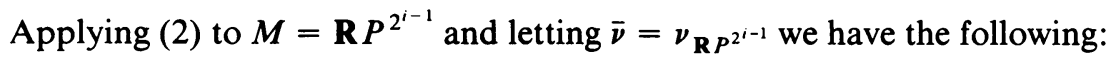

$$
\exists \text { a map } \phi: \Sigma^{2^{i-1}} T(\bar{\nu}) \rightarrow D_{2^{i-1}} \text { such that } \phi^{*}\left(u_{2^{i-1}}\right)=U .
$$

We have $\Sigma^{2^{i-1}} T(\bar{\nu}) \simeq \Sigma^{2^{i}+1} P_{-2^{i-1}-1}^{-2}[4,10]$. Since the Stiefel-Whitney class $\omega_{2^{i-1}-1}(\bar{\nu})$ is nonzero, it follows that $\mathrm{Sq}^{2^{i-1}-1}(U) \neq 0$, which is the top class of $H^{*}\left(\Sigma^{2^{i}+1} P_{-2^{i-1}-1}^{-2}\right)$. Let $v=\operatorname{Sq}^{2^{i-1}-1}\left(u_{2^{i-1}}\right) \in H^{2^{i}-1}\left(D_{2^{i-1}}\right)=\mathbf{Z}_{2}$. From (3) we see that

$\exists$ a map $\phi: \Sigma^{2^{i}+1} P_{-2^{i-1}-1}^{-2} \rightarrow D_{2^{i-1}}$ such that $\phi^{*}(v) \neq 0$ in $H^{2^{i}-1}\left(\Sigma^{2^{i}+1} P_{-2^{i-1}-2}^{-2}\right)=\mathbf{Z}_{2}$. 
Mahowald also shows in [11], and this is crucial, that

$\exists$ a stable map $\tilde{\eta}: \Omega^{2} S_{+}^{3} \simeq \mathrm{V}_{k \geqslant 0} D_{k} \rightarrow S^{0}$ such that

$$
\mathrm{Sq}^{2^{i}}: H^{0}\left(S^{0} \cup_{\tilde{\eta} \mid D_{2^{i-1}}} C D_{2^{i-1}}\right)=\mathbf{Z}_{2} \rightarrow H^{2^{i}}\left(S^{0} \cup_{\tilde{\eta} \mid D_{2^{i-1}}} C D_{2^{i-1}}\right)=\mathbf{Z}_{2}
$$

is nonzero for each $i \geqslant 1$.

Proposition 2(a) follows by (4) and (5).

We remark that in [11] Mahowald only considers $\Omega^{2} S^{9}$ to get a result analogous to (5). But his argument also works for $\Omega^{2} S^{3}$.

Our main work is to show Proposition 2(b). Let $\eta, \nu$ and $\sigma$ be the Hopf classes in $\pi_{1}^{s}, \pi_{3}^{s}$ and $\pi_{7}^{s}$, respectively [15]. H. Toda has shown [15] that $\left\langle\nu, \eta, \sigma^{2}\right\rangle$ consists of a single element $\bar{\sigma}$ which has order 2. May observed [12] that $\bar{\sigma}$ is detected by $c_{1}$. From the definition of the Toda bracket $\left\langle\nu, \eta, \sigma^{2}\right\rangle$ we see that, for any extension $\tilde{\nu}$ : $S^{1} \cup_{\eta} e^{3} \rightarrow S^{-2}$ of $\nu: S^{1} \rightarrow S^{-2}$ and any coextension $\overline{\sigma^{2}}: S^{17} \rightarrow S^{1} \cup_{\eta} e^{3}$ of $\sigma^{2}$ : $S^{17} \rightarrow S^{3}$

the composite $S^{17} \stackrel{\overline{\sigma^{2}}}{\rightarrow} S^{1} \cup_{\eta} e^{3} \stackrel{\tilde{\nu}}{\rightarrow} S^{-2}$ is always $\bar{\sigma}$ and is detected by $c_{1}$.

Consider $\Sigma^{-1} P_{1}^{4}=S^{0} \cup_{2 \iota} e^{1} \cup e^{2} \cup_{2 \iota} e^{3}$ which is the mapping cone of $S^{2} \stackrel{(\bar{\eta}, 2 \iota)}{\rightarrow}\left(S^{0}\right.$ $\left.\cup_{2 \iota} e^{1}\right) \vee S^{2}$, where $\bar{\eta}$ is a coextension of $\eta: S^{2} \rightarrow S^{1}$. There is an obvious map

$$
\Sigma^{-1} P_{1}^{4} \stackrel{q}{\rightarrow} S^{1} \cup_{\eta} e^{3}
$$

obtained by pinching $S^{0} \cup e^{2}=S^{0} \vee S^{2}$ to a point. Toda also shows in [15] that $\left\langle 2 \iota, \eta, \sigma^{2}\right\rangle=\{0\}$ and $2 \sigma^{2}=0$. These imply that there is a map $\overline{\overline{\sigma^{2}}}: S^{17} \rightarrow \Sigma^{-1} P_{1}^{4}$ such that

$$
\text { the composite } S^{17} \stackrel{\overline{\overline{\sigma^{2}}}}{\rightarrow} \Sigma^{-1} P_{1}^{4} \stackrel{q}{\rightarrow} S^{1} \cup_{\eta} e^{3} \text { is a } \overline{\sigma^{2}} \text {. }
$$

We will show that there is a map $\bar{g}: \Sigma^{-1} P_{1}^{4} \rightarrow P_{-2^{i-1}-1}^{-2}$ such that

$$
\text { the composite } \Sigma^{-1} P_{1}^{4} \stackrel{\bar{g}}{\rightarrow} P_{-2^{i-1}-1}^{-2} \stackrel{p}{\rightarrow} S^{-2} \text { is equal to the com- }
$$

posite $\Sigma^{-1} P_{1}^{4} \stackrel{q}{\rightarrow} S^{1} \cup_{\eta} e^{3} \stackrel{\tilde{\nu}}{\rightarrow} S^{-2}$ for some $\tilde{\nu}$.

(6), (7) and (8) imply Proposition 2(b) by taking $g=\bar{g} \circ \overline{\overline{\sigma^{2}}}$.

To show (8) consider the cofibration sequence

$$
\Sigma^{-1} P_{-2^{i-1}-1}^{-1} \rightarrow \Sigma^{-1} P_{-2^{i-1}-1}^{4} \rightarrow \Sigma^{-1} P_{0}^{4} \stackrel{\tilde{g}}{\rightarrow} P_{-2^{i-1}-1}^{-1}
$$

induced by the inclusion $\Sigma^{-1} P_{-2^{i-1}-1}^{-1} \rightarrow \Sigma^{-1} P_{-2^{i-1}-1}^{4}$. Here $\tilde{g}$ is not uniquely determined; it can be altered, say, by any self-homotopy equivalence $\Sigma^{-1} P_{0}^{4} \rightarrow \Sigma^{-1} P_{0}^{4}$. I. M. James shows in [10] that $P_{-2^{i-1}-1}^{-1} \simeq P_{-2^{i-1}-1}^{2^{2}} \vee S^{-1}$ and $\Sigma^{-1} P_{0}^{4} \simeq S^{-1} \vee \Sigma^{-1} P_{1}^{4}$. Consider the composite

$$
\bar{g}: \Sigma^{-1} P_{1}^{4} \stackrel{\tilde{g} \mid \Sigma^{-1} P_{1}^{4}}{\rightarrow} P_{-2^{i-1}-1}^{-1} \simeq P_{-2^{i-1}-1}^{-2} \vee S^{-1} \rightarrow P_{-2^{i-1}-1}^{-2}
$$


where the second map is the projection. We will show that there is a choice of $\tilde{g}$ such that

the composite $\Sigma^{-1} P_{1}^{4} \stackrel{\bar{g}}{\rightarrow} P_{-2^{i-1}-1}^{-2} \stackrel{p}{\rightarrow} S^{-2}$ is zero on the bottom cell of $\Sigma^{-1} P_{1}^{4}$.

Granting (9) we see that, since $\pi_{4}^{s}=0, p \circ \bar{g}$ is trivial on the subcomplex $S^{0} \cup e^{2}=$ $S^{0} \vee S^{2}$ and therefore factorizes through $S^{1} \cup_{\eta} e^{3}$, and it is easy to see that the factorization map $S^{1} \cup_{\eta} e^{3} \rightarrow S^{-2}$ is a $\tilde{\nu}$. Thus $\bar{g}$ satisfies (8).

Suppose $\lambda=p \circ\left(\bar{g} \mid S^{0}\right): S^{0} \rightarrow S^{-2}$ is nonzero; so $\lambda=\eta^{2}$. It is easy to see that the composite

$$
S^{-1} \stackrel{\tilde{g} \mid S^{-1}}{\rightarrow} P_{-2^{i-1}-1}^{-1} \simeq P_{-2^{i-1}-1}^{-2} \vee S^{-1} \rightarrow P_{-2^{i-1}-1}^{-2} \stackrel{p}{\rightarrow} S^{-2}
$$

is $\eta$. We have $2 \nu \in\langle\eta, 2 \iota, \eta\rangle[15]$. Hence there is a map $\eta^{\prime}: \Sigma^{-1} P_{1}^{4} \rightarrow S^{-1}$ which, when restricted to $S^{0}$, is $\eta$. Consider the map

$$
\Sigma^{-1} P_{0}^{4}=S^{-1} \vee \Sigma^{-1} P_{1}^{4} \stackrel{\alpha=\left[\begin{array}{cc}
1 & \eta^{\prime} \\
0 & 1
\end{array}\right]}{\longrightarrow} \Sigma^{-1} P_{0}^{4}=S^{-1} \vee \Sigma^{-1} P_{1}^{4} .
$$

It is clear that $\alpha$ is a self-homotopy equivalence. Let $\tilde{g}^{\prime}=\tilde{g} \circ \alpha: \Sigma^{-1} P_{0}^{4} \rightarrow P_{-2^{i-1}-1}^{-1}$. Then the corresponding $\bar{g}^{\prime}$ satisfies (9). This completes the proof.

\section{REFERENCES}

1. J. F. Adams, On the structure and applications of the Steenrod algebra, Comment. Math. Helv. 32 (1985), 180-214.

2. On the non-existence of elements of Hopf invariant one, Ann. of Math. (2) 72 (1960), 20-104.

3. Operations of the n-th kind in K-theory and what we don' $t$ know about $\mathbf{R} P^{\infty}$, London Math. Soc. Lecture Note Ser. 11 (1974), 1-9.

4. M. F. Atiyah, Thom complexes, Proc. London Math. Soc. (3) 11 (1961), 291-310.

5. E. H. Brown, Jr. and S. Gitler, $A$ spectrum whose cohomology is a certain cyclic module over the Steenrod algebra, Topology 12 (1973), 283-295.

6. E. H. Brown, Jr. and F. P. Peterson, On the stable decomposition of $\Omega^{2} S^{r+2}$, Trans. Amer. Math. Soc. 243 (1978), 287-298.

7. R. Bruner, An infinite family in $\pi_{*} S^{0}$ derived from Mahowald's $\eta_{j}$ family, Proc. Amer. Math. Soc. 82 (1981), 637-639.

8. R. L. Cohen, J. D. S. Jones and M. E. Mahowald, The Kervaire invariant of immersions, Ivent. Math. (to appear).

9. D. M. Davis, An infinite family in the cohomology of the Steenrod algebra, J. Pure Appl. Algebra 21 (1981), 145-150.

10. I. M. James, Spaces associated with Stiefel manifolds, Proc. London Math. Soc. (3) 9 (1959), 115-140.

11. M. E. Mahowald, $A$ new infinite family in ${ }_{2} \pi_{*}^{s}$, Topology 16 (1977), 249-256.

12. J. P. May, The cohomologv of restricted Lie algebras and Hopf algebras; application to the Steenrod algebra, Dissertation, Princeton Univ., 1964.

13. V. P. Snaith, $A$ stable decomposition for $\Omega^{n} S^{n} X$, J. London Math. Soc. (2) 2 (1974), 577-583.

14. M. C. Tangora, On the cohomology of the Steenrod algebra, Math. Z. 116 (1970), 18-64.

15. H. Toda, Composition methods in homotopy groups of spheres, Ann. of Math. Stud., no. 49, Princeton Univ. Press, Princeton, N. J., 1962.

School of Mathematics, Institute for Advanced Study, Princeton, New Jersey 08540

Department of Mathematics, NorthWestern University, Evanston, Illinois 60201

Department of Mathematics, National Tsing Hua University, Hsinchu (300), Taiwan 